\title{
NARROW-BAND IMAGING OF THE NUCLEAR REGION OF THE SEYFERT GALAXY NGC1068
}

\author{
T.ISHIGAKI, H.OHTANI, T.HAYASHI, S.OZAKI, T.HATTORI AND \\ H.SUGAI \\ Department of Astronomy, Kyoto University \\ Sakyo-ku, Kyoto 606-01, Japan \\ M.SASAKI \\ Shimonoseki City University \\ Daigaku-cho, Shimonoseki 751, Japan \\ AND \\ K.AOKI, M.YOSHIDA AND E.WATANABE \\ National Astronomical Observatory \\ Mitaka, Tokyo 181, Japan
}

\section{Observation and Results}

A narrow-band imaging observation of the Seyfert galaxy NGC1068 was made in the Fabry-Perot mode of the Kyoto 3D Spectrograph attached to the $188 \mathrm{~cm}$ telescope of the Okayama Astrophysical Observatory. We observed at wavelengths of $\mathrm{H} \alpha,[\mathrm{S} \mathrm{II}] \lambda 6716$, [S II] $]$ 6731 and adjacent continua of the respective lines with a "tunable filter", i.e. a gap-scanning etalon with a spectral resolution of $20 \AA$.

Line-flux maps of $\mathrm{H} \alpha$ and [S II] $\lambda 6716+\lambda 6731$ are shown in Figure $1 \mathrm{a}$ and $1 \mathrm{~b}$ respectively. The latter is the first [S II] image of NGC1068 as far as we know. It should be noticed that the distribution of [S II] emission around the nucleus is more extended in the direction of E-W than the distribution of $\mathrm{H} \alpha$ emission. This feature is more clearly seen in the [S II]/ $\mathrm{H} \alpha$ map shown in Figure 1c. In this figure, regions with larger [S II]/H $\alpha$ ratio have bipolar morphology along E-W direction. At $10^{\prime \prime} \mathrm{SW}$ of the nucleus, an arc-like structure is also recognized. This arc-like structure is located along the radio axis and delineates the $\mathrm{SW}$ radio lobe. Close comparison of the [S II] $/ \mathrm{H} \alpha$ map with the $[\mathrm{O} \mathrm{III}] / \mathrm{H} \alpha$ map (Pogge 1988) reveals that $[\mathrm{S} \mathrm{II}] / \mathrm{H} \alpha$ 
a) $\mathrm{Ha}$

b) [SII]

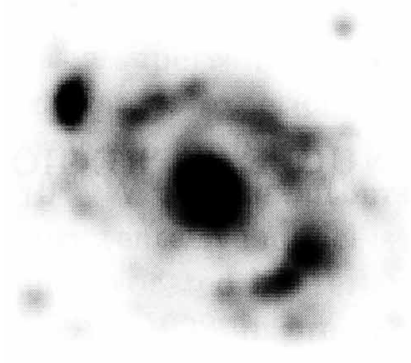

c) $[\mathrm{SII}] / \mathrm{Ha}$

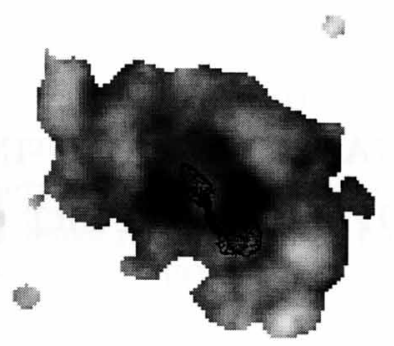

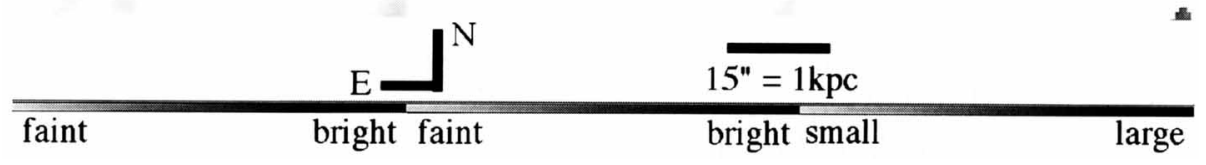

Figure 1. a) $\mathrm{H} \alpha$ image. b) [SII] image. c) [SII] $/ \mathrm{H} \alpha$ map with the radio continuum contours of Wilson \& Ulvestad (1983) superposed.

ratio shows an anti-correlated distribution with $[\mathrm{O} \mathrm{III}] / \mathrm{H} \alpha$ ratio around the nucleus. We compared the $[\mathrm{S} \mathrm{II}] / \mathrm{H} \alpha$ map with the $\mathrm{CO}$ emission (Helfer \& Blitz 1995) to find that the arc-like structure at $10^{\prime \prime} \mathrm{SW}$ lies along the inner edge of a $\mathrm{CO}$ arm.

\section{Discussion}

The present observation has shown that outside the ionization cone are some appreciable amounts of ionized gases which are characterized by large [S II] $/ \mathrm{H} \alpha$ and small $[\mathrm{O} \mathrm{III}] / \mathrm{H} \alpha$. These gases may be ionized by scattered light of the nuclear continuum and/or the continuum attenuated by obscuring material. The anti-correlation between $[\mathrm{S} \mathrm{II}] / \mathrm{H} \alpha$ and $[\mathrm{O} \mathrm{III}] / \mathrm{H} \alpha$ could be explained by the variation of ionization parameter.

It is likely that the arc-like structure is an ionization front produced along the inner surface of the molecular arm facing directly to the nucleus. Alternatively, the coincidence of the arc along the leading edge of the radio lobe may imply that this structure corresponds shock heated gases produced by the radio jet. This may be supported by the fact that the velocity gradients of ionized gases suddenly change at this location (Kaneko et al. 1992).

\section{References}

Helfer, T.T. and Blitz, L. (1995) $A p J, 450,90$

Kaneko, N., Satoh, T., Toyama, K., Sasaki, M., Nishimura, M. and Yamamoto, M. (1992) $A J, 103,422$

Pogge, R. W. (1988) ApJ, 328, 519

Wilson, A. S. and Ulvestad, J. S. (1983) $A p J, 275,8$ 